\title{
Bloch-like oscillations in the Peyrard-Bishop-Holstein model
}

\author{
E. Díaz, R. P. A. Lima, and F. Domínguez-Adame \\ GISC, Departamento de Física de Materiales, Universidad Complutense, E-28040 Madrid, Spain \\ (Received 18 June 2008; revised manuscript received 7 September 2008; published 9 October 2008)
}

\begin{abstract}
The Peyrard-Bishop-Holstein model has been previously introduced as an appropriate framework for the description of polaronic effects for charge migration in DNA. We study numerically the Peyrard-BishopHolstein model when the charge carrier is also subjected to an applied uniform electric field. We find that the polaron undergoes coherent oscillations when the electric field is applied along the stacking direction. The frequency of the oscillations is the same as in the rigid lattice (Bloch frequency) provided that the carrier-lattice coupling is not large. Increasing the coupling the single peak of the Fourier spectrum splits into side peaks around the Bloch frequency.
\end{abstract}

DOI: 10.1103/PhysRevB.78.134303

PACS number(s): 71.38.-k, 72.10.-d, 87.15.A-

\section{INTRODUCTION}

Recent advances in molecular electronics allow for controlled manipulation of DNA molecules for electric transport measurements possible. ${ }^{1,2}$ Experiments on electric transport through dry and wet DNA molecules revealed a variety of results ranging from proximity-induced superconducting, ${ }^{3}$ Ohmic-type, ${ }^{4-7}$ semiconducting, ${ }^{8-12}$ and insulating ${ }^{13,14}$ behaviors. It is believed that the environment of the molecule, the sequence of nucleotides, and lead effects play an outstanding role in the observed differences. In addition, carrier coupling to low-frequency vibrational modes may give rise to a significant improvement of the conductance. ${ }^{15}$ Recent experiments in poly $(\mathrm{G})$-poly $(\mathrm{C})$ and poly(A)-poly(T) DNA molecules in solution suggest that they may act as nanowires supporting high electrical current under moderate bias. ${ }^{9}$

The nucleotide sequence in synthetic poly $(\mathrm{G})$-poly $(\mathrm{C})$ and poly(A)-poly(T) DNA molecules is periodic. Consequently they can be regarded as artificial crystals that can carry electric current when they are subjected to an external bias. Electrons in periodic potentials subjected to an applied electric field present dynamic localization ${ }^{16}$ and may undergo coherent oscillations. ${ }^{17,18}$ They perform a periodic motion, in real and in $k$ spaces, known as Bloch oscillations (BOs). ${ }^{19,20}$ From semiclassical arguments it can be shown that BOs are characterized by a time period $\tau_{B}=2 \pi \hbar / e F a$ and an amplitude $A_{B}=W / e F$, where $-e$ is the electron charge, $F$ is the applied electric field, $a$ denotes the spatial period of the potential, and $W$ stands for the band width. BOs were observed as coherent oscillations of electronic wave packets in semiconductor superlattices $^{21,22}$ (see Ref. 23 for an overview). These oscillations are related to the wave dynamics of particles, and therefore they can be observed in almost any coherent motion of waves in tilted periodic potentials. Thus, they were later detected as a periodic motion of ensembles of ultracold atoms ${ }^{24,25}$ and Bose-Einstein condensates ${ }^{26}$ in tilted optical lattices.

BOs persist until electrons lose their phase coherence through scattering processes. The scattering time $\tau$ must be larger than the Bloch period $\tau_{B}$ and therefore the electric field must exceed a critical value. ${ }^{27}$ Inelastic scattering by phonons, deviations from perfect periodicity due to imperfections, intraband scattering, and scattering by impurities severely reduce the quantum coherence required for the observation of BOs. Even in the most favorable experimental conditions, $\tau$ is not much larger that $\tau_{B}$ and only a few BOs are usually observed. DNA molecules largely differ from inorganic solids because vibrations of nucleotides are significant and could rapidly degrade the electron quantum coherence. Lakhno and Fialko ${ }^{28}$ found that homogeneous nucleotide sequences display Bloch oscillations under applied electric fields even if electron-lattice coupling is taken into account provided that temperature is low. Their model, however, considers uncoupled nucleotides and small (harmonic) vibrations.

In this work we focus on a more realistic model of carrier dynamics in a deformable DNA molecule subjected to an external applied electric field, which is assumed uniform hereafter. To this end, here we will take advantage of the accurate description of the nucleotide dynamics and its effects on electric transport provided by the Peyrard-BishopHolstein (PBH) model. ${ }^{29-31}$ The lattice dynamics is nonlinear, beyond the harmonic approximation, and it allows for a better description of large-amplitude vibrations of nucleotides coupled along each strand. Our aim here is to investigate whether the polaron performs sustainable BOs in biased DNA molecules described by the PBH model.

\section{MODEL}

In the PBH model the carrier is treated quantum mechanically, within the framework of the tight-binding approximation, and the lattice dynamics is taken into account classically. For simplicity we will restrict ourselves to the electronvibration coupling in a single strand. Thus, the Schrödinger equation for the carrier is given by ${ }^{31}$

$$
i \hbar \frac{d \psi_{n}}{d t}=-U n \psi_{n}-T\left(\psi_{n+1}+\psi_{n-1}\right)+\chi y_{n} \psi_{n},
$$

where $\psi_{n}$ is the probability amplitude for the charge carrier located at the $n$th nucleotide. The parameter $U=e F a$ is the potential-energy drop across the period of the lattice ( $a$ $=3.4 \AA$ in DNA). The hopping is restricted to nearestneighbor nucleotides and its magnitude is given by $-T$. The last term in Eq. (1) describes the carrier-vibration coupling 
through the constant $\chi$ and the displacement $y_{n}$ of the $n$th nucleotide from its equilibrium position.

Newton's equations of motion for the displacement $y_{n}$ become

$$
m \frac{d^{2} y_{n}}{d t^{2}}=-V_{M}^{\prime}\left(y_{n}\right)-W^{\prime}\left(y_{n}, y_{n-1}\right)-W^{\prime}\left(y_{n}, y_{n+1}\right)-\chi\left|\psi_{n}\right|^{2},
$$

where $m$ is the nucleotide mass and the prime indicates differentiation with respect to $y_{n}$. The Morse potential

$$
V_{M}\left(y_{n}\right)=V_{0}\left(e^{-\alpha y_{n}}-1\right)^{2}
$$

takes into account the anharmonic interaction between complementary base pairs as well as the interaction with the sugar-phosphate backbone. The interaction between nearestneighbor nucleotides along the staking direction is described by the potential ${ }^{31}$

$$
W\left(y_{n}, y_{n-1}\right)=\frac{k}{4}\left(2+e^{-\beta\left(y_{n}+y_{n-1}\right)}\right)\left(y_{n}-y_{n-1}\right)^{2} .
$$

The values of the potential parameters are given by $m$ $=300 \mathrm{amu}, V_{0}=0.04 \mathrm{eV}, \alpha=4.45 \AA^{-1}, k=0.04 \mathrm{eV} / \AA^{2}, \beta$ $=0.35 \AA^{-1}$, and $T=0.1 \mathrm{eV} .^{31}$ It should be noted that $a b$ initio estimations of the coupling constant $\chi$ are scarce and the results are strongly dependent on the sequence and number of nucleotides. ${ }^{32}$ Therefore, we will vary its magnitude in the numerical simulations to study the effects on the carrier dynamics.

\section{RESULTS}

In order to study the time evolution of the polaron in the biased lattice, we numerically solved the set of nonlinear equations (1) and (2) using a Runge-Kutta method of fourth order considering hereafter the homopolymer DNA case under rigid boundary conditions. To obtain the initial polaron state we follow the procedure given in Ref. 30 in an unbiased lattice $(U=0)$, including a dissipative term of the form $-\gamma m d y_{n} / d t$ in Eq. (2) with $\gamma=50 \mathrm{THz}$. Once the stationary solution is found, it is then taken as the initial condition at $t=0$ to solve Eqs. (1) and (2) with $U \neq 0$, neglecting dissipation. The initial polaron state used in our simulations is shown in Fig. 1 for different values of $\chi$ and $N=500$. It is to be noticed that the motion of the polaron in a biased lattice with dissipation is uniform with constant speed. ${ }^{32}$ Therefore, dissipation destroys the quantum coherence required for the observation of BOs. In a real situation, one expects that BOs could be observed at short times after the initial excitation. This is the regime in which we are interested in this work as we discussed in Sec. IV in more detail.

The time-domain evolution of the carrier wave function provides detailed information about what happens after the initial excitation of the system. Figure 2(a) shows the wave function in a lattice of $N=1000$ sites as a function of position and time for $\chi=0.1 \mathrm{eV} / \AA$ and $F=3.0 \mathrm{mV} / \AA$. The carrier displays clear signatures of a coherent oscillation with small distortion of its initial shape. The observed frequency is about $15.52 \mathrm{THz}$, which is fairly close to the semiclassical

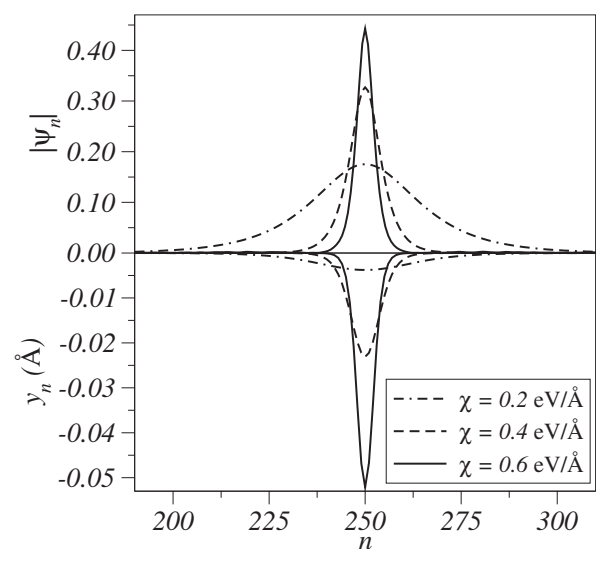

FIG. 1. Carrier wave function (upper panel) and lattice displacement (lower panel) at $t=0$ for different values of $\chi$ and $N=500$.

value $\omega_{B}=e F a / \hbar=15.502 \mathrm{THz}$. Figure 2(b) shows the corresponding lattice displacement. The lattice also performs oscillations but with a main frequency of $7.22 \mathrm{THz}$ for the same parameters used in Fig. 2(a). This frequency is not related to the Bloch frequency $\omega_{B}$. On the contrary, it is close to the Morse frequency $\omega_{M}$, namely, the harmonic frequency of the small amplitude oscillations of a particle with mass $m$ around the minimum of the Morse potential (3). This fre-
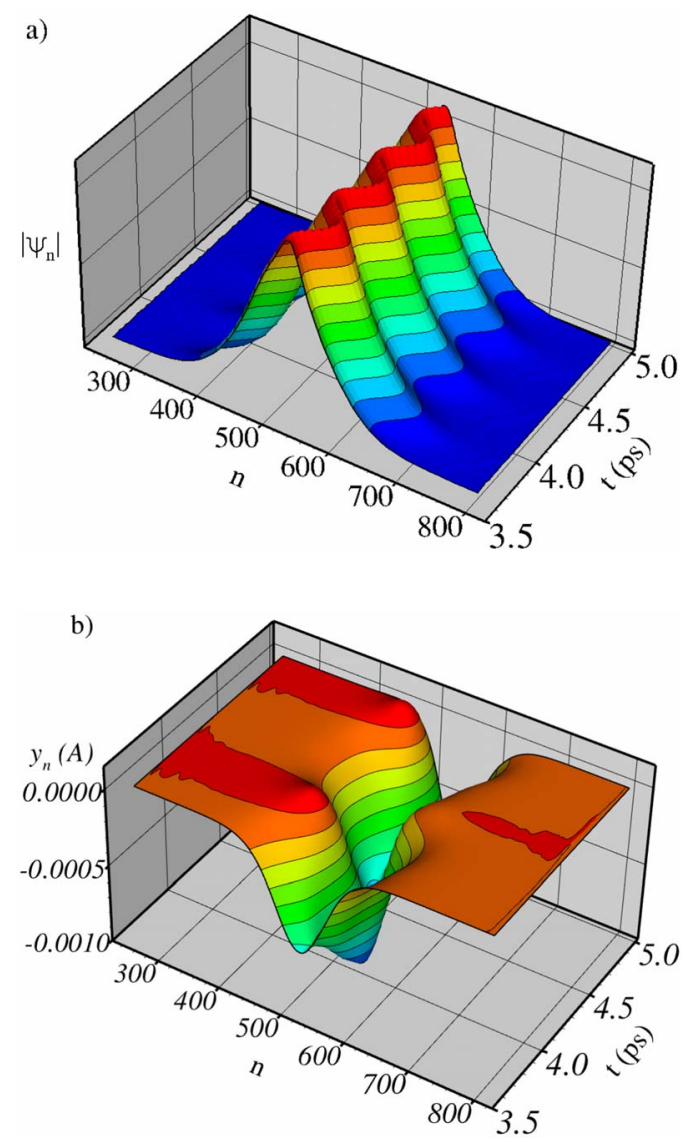

FIG. 2. (Color online) (a) Carrier wave function and (b) lattice displacement as a function of position and time in a lattice of $N$ $=1000$ sites for $\chi=0.1 \mathrm{eV} / \AA$ and $F=3.0 \mathrm{mV} / \AA$ showing their oscillatory behavior. 

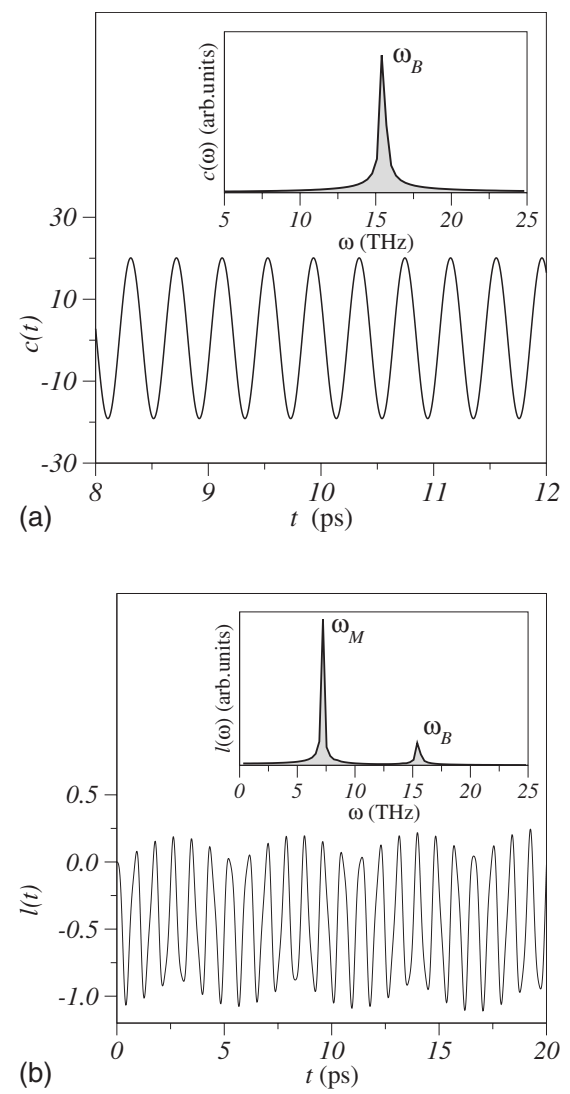

FIG. 3. (a) Centroid of the carrier wave function and (b) $l(t)$ as a function of time in a lattice of $N=1000$ sites for $\chi=0.1 \mathrm{eV} / \AA$ and $F=3.0 \mathrm{mV} / \AA$. The insets show the corresponding Fourier transform.

quency is found to be $\omega_{M}=\alpha \sqrt{2 V_{0} / m}=7.138 \mathrm{THz}$ for the set of parameters used in the simulation. Therefore, we come to the conclusion that the oscillations of the lattice and the carrier are almost decoupled at least at small applied fields. It is to be noticed that for larger fields our solutions become unstable especially for small values of the carrier-vibration coupling $\chi$. This might be produced by the numerical method used to solve the set of nonlinear equations. However it could also be due to some intrinsic instability of the problem. Indeed in Ref. 32 the authors already claimed the existence of a maximum electric field that the polaron can tolerate.

The value of the frequencies involved in the polaron dynamics under an external bias can be determined accurately by calculating the Fourier transform of the centroid $c(t)$ of the carrier wave function

$$
c(t)=x(t)-x(0), \quad x(t)=\sum_{n=1}^{N} n\left|\psi_{n}(t)\right|^{2} .
$$

Similarly for the lattice displacements we define the dimensionless magnitude $l(t)$ as follows:

$$
l(t)=\xi(t)-\xi(0), \quad \xi(t)=\sum_{n=1}^{N} n y_{n}(t) / a .
$$

Figure 3 displays the calculated $c(t)$ and $l(t)$ in a lattice of

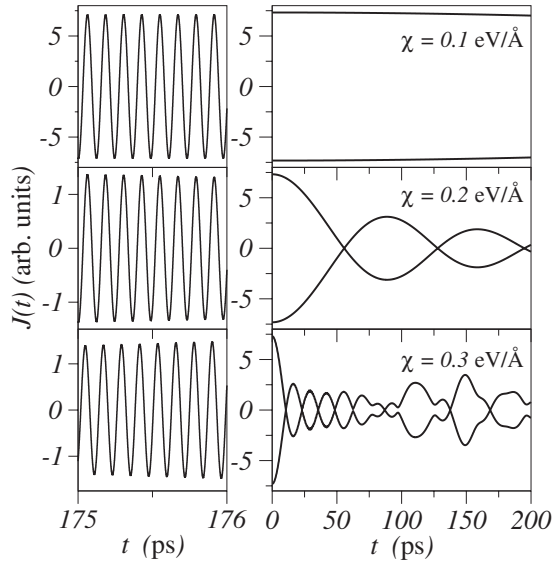

FIG. 4. Current density as a function of time when $F$ $=10 \mathrm{mV} / \AA$ for different values of the coupling constant, namely, $\chi=0.1,0.2,0.3 \mathrm{eV} / \AA$ from top to bottom. The left panels show the oscillatory behavior over a short-time interval. The right panels only show the envelope of the current and over a much longer time interval.

$N=1000$ sites for $\chi=0.1 \mathrm{eV} / \AA$ and $F=3.0 \mathrm{mV} / \AA$. As expected, the centroid $c(t)$ performs a harmonic motion with frequency equal to $\omega_{B}=e F a / \hbar=15.52 \mathrm{THz}$ within the numerical uncertainty [see the inset in Fig. 3(a)]. However, the lattice displacements display a more complex motion, as seen in Fig. 3(b). The main frequency is about $7.22 \mathrm{THz}$, which is close to the Morse frequency $\omega_{M}=7.138 \mathrm{THz}$ discussed above. Furthermore, we observe the occurrence of a smaller peak at the Bloch frequency [see the inset in Fig. $3(b)$. In spite of the complex behavior of the lattice displacements in the frequency domain, let us stress that the carrier motion is characterized by a single frequency, namely, the Bloch frequency.

To gain additional insight into the polaron dynamics in the biased lattice, we calculate the average current density $J(t)$ through the bases ${ }^{31}$

$$
J(t)=\frac{\hbar e}{m_{e} N a^{2}} \sum_{n=1}^{N} \operatorname{Im}\left[\psi_{n}^{*}\left(\psi_{n+1}-\psi_{n-1}\right)\right],
$$

where $m_{e}$ is the mass of the carrier. Typical results of our simulations are collected in Fig. 4. The left panels show the time-dependent current density over a short time interval when $F=10 \mathrm{mV} / \AA$ and several values of the coupling constant $\chi=0.1,0.2,0.3 \mathrm{eV} / \AA$. The current density displays a well-defined oscillatory behavior. Remarkably the time period matches almost perfectly the semiclassical value $\tau_{B}$ $=2 \pi \hbar / e F a=0.122 \mathrm{ps}$ in the rigid lattice for the three coupling constants shown in Fig. 4. The right panels show the envelope of the curve $J(t)$ over a much larger time interval for the same values of the coupling constant. The increase in the coupling constant leads to a faster modulation of the current density but, remarkably, the oscillations do not decay on time. We will comment about the relevance of this finding in Sec. IV.

In order to clarify which frequencies are playing a major role in the polaron dynamics, the Fourier transform of the 


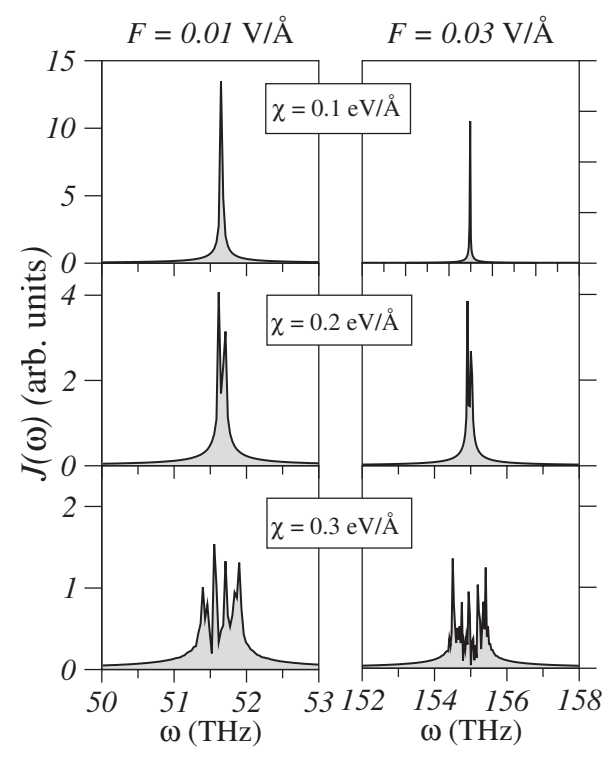

FIG. 5. Fourier transform of the current density $J(t)$ for $F$ $=10 \mathrm{mV} / \AA$ (left panels) and $F=30 \mathrm{mV} / \AA$ (right panels) and three different values of the coupling constant, namely, $\chi$ $=0.1,0.2,0.3 \mathrm{eV} / \AA$ from top to bottom.

average current density was calculated for several values of the coupling constant $\chi$ and electric field $F$ (see Fig. 5). For small coupling constant, the Fourier spectrum transforms in a single peak at the semiclassical value of the Bloch frequency $\omega_{B}=2 \pi / \tau_{B} \quad\left(\omega_{B}=51.67\right.$ and $155.02 \mathrm{THz}$ for $F=10$ and $30 \mathrm{mV} / \AA$, respectively). On increasing the coupling constant $\chi$, side peaks around $\omega_{B}$ appear, as seen in the lower panels of Fig. 5. But even in the case of a broad Fourier spectrum $(\chi=0.3 \mathrm{eV} / \AA$ in Fig. 5), quasiharmonic oscillations are observed over time intervals larger than $\tau_{B}$ (see lower right panel of Fig. 4).

The occurrence of satellite peaks around the Bloch frequency in the Fourier spectrum (Fig. 5) can be understood as follows. The diagonal term in Eq. (1) is given by $(-U n$ $\left.+\chi y_{n}\right) \psi_{n}$. Expanding the lattice displacement around the site with maximum electron amplitude $s(t)$ up to first order, one gets $y_{n}(t) \simeq y_{s}(t)+\rho(t)[n-s(t)]$. Consequently, the diagonal term is given approximately by $\left[-U_{\text {eff }}(t) n+a(t)\right] \psi_{n}$ with $a(t)=\chi\left[y_{s}(t)-\rho(t) s(t)\right]$ and $U_{\text {eff }}(t)=U-\chi \rho(t)$. Therefore, there is a local contribution to the external field coming from the deformation of the lattice. This extra contribution $-\chi \rho(t)$ depends on time, giving rise to a complex-frequency spectrum.

As mentioned in Sec. I, scattering by defects, charges, and phonons destroys the phase coherence at times larger than the scattering time $\tau$. Therefore, an initially excited polaron performs a coherent oscillatory motion provided that $\tau_{B}<\tau$.
The scattering time $\tau$ depends on temperature and Lakhno and Fialko ${ }^{28}$ estimated that $\tau \sim T^{-2.3}$. This estimation yields the temperature $T_{\min }$ below which BOs take place at a given magnitude of the electric field, $T_{\min } \simeq 0.45 T_{0}\left(\omega_{B} \tau_{0}\right)^{1 / 2.3}$. Here $\tau_{0} \simeq 6.4 \mathrm{fs}$ is the scattering time at $T_{0}=300 \mathrm{~K} .{ }^{28}$ At the lowest field considered in this work, $F=3.0 \mathrm{mV} / \AA, T_{\min }$ $=50 \mathrm{~K}$ is obtained. Larger values of the electric field raise this threshold temperature. At much larger time $t \gg \tau$, the polaron moves classically with uniform velocity. ${ }^{32}$ We thus claim that our results should be relevant at short times after the initial excitation and lattice relaxation.

\section{CONCLUSIONS}

We investigated the polaron dynamics within the PeyrardBishop-Holstein model in the presence of a uniform electric field. When the coupling of the charge carrier and the lattice is neglected, it is well known that coherent and harmonic oscillations arise, namely, BOs. ${ }^{17,18}$ The frequency of the BOs is estimated from a semiclassical approach to be $\omega_{B}$ $=e F a / \hbar$. Remarkably, we have found that the polaron of the unbiased lattice also displays coherent oscillations when the bias is switched on. The oscillations detected in the current density are quasiharmonic over short time intervals with a frequency that matched the Bloch frequency $\omega_{B}$ for the corresponding applied field. With the parameters used in DNA, when the potential drop across a single period is about the electron bandwidth, i.e., $U \sim 4 T=0.4 \mathrm{eV}$ the Bloch frequency is $\omega_{B} \sim 607.9 \mathrm{THz}$, making DNA a potential candidate for electronic applications in the terahertz range. Increasing the coupling constant leads to the occurrence of side peaks in the Fourier transform around the Bloch frequency. Nevertheless, the current density still displays a well-defined oscillatory pattern with a main frequency close to the Bloch frequency.

From our analysis it becomes clear that the coupling parameter $\chi$ should be as small as possible. Recently, Berashevich et al. ${ }^{32}$ have found that $\chi$ is smaller in poly(dA)poly $(\mathrm{dT})$ than in poly $(\mathrm{dG})-\operatorname{poly}(\mathrm{dC})$ synthetic DNA. In addition, they found a slight decrease in the coupling constant upon increasing the number of nucleotides. Thus, for $N=4$ they get $\chi=0.3 \mathrm{eV} / \AA$. We have found that for such value of the coupling constant, coherent oscillations of the polaron can arise.

\section{ACKNOWLEDGMENTS}

The authors thank E. Maciá and A. V. Malyshev for helpful conversations. This work was supported by MEC (Project MOSAICO) and BSCH-UCM (Project No. PR34/07-15916). R.P.A.L. acknowledges support by MEC through the Juan de la Cierva program. 
${ }^{1}$ D. Porath, N. Lapidot, and J. Gómez-Herrero, Introducing Molecular Electronics, edited by G. Cuniberti, G. Fagas, and K. Richter (Springer, Berlin, 2005).

${ }^{2}$ G. Cuniberti, E. Maciá, A. Rodríguez, and R. A. Röemer, Charge Migration in DNA, edited by T. Chakraborty (Springer, Berlin, 2007).

${ }^{3}$ A. Yu. Kasumov, M. Kociak, S. Guéron, B. Reulet, V. T. Volkov, D. V. Klinov, and H. Bouchiat, Science 291, 280 (2001).

${ }^{4}$ Y. Okahata, T. Kobayashi, K. Tanaka, and M. J. Shimomura, J. Am. Chem. Soc. 120, 6165 (1998).

${ }^{5}$ H. W. Fink and C. Schönenberger, Nature (London) 398, 407 (1999).

${ }^{6}$ A. Rakitin, P. Aich, C. Papadopoulos, Yu. Kobzar, A. S. Vedeneev, J. S. Lee, and J. M. Xu, Phys. Rev. Lett. 86, 3670 (2001).

${ }^{7}$ O. Legrand, D. Côte, and U. Bockelmann, Phys. Rev. E 73, 031925 (2006).

${ }^{8}$ D. Porath, A. Bezryadin, S. de Vries, and C. Dekker, Nature (London) 403, 635 (2000).

${ }^{9}$ K.-H. Yoo, D. H. Ha, J.-O. Lee, J. W. Park, J. Kim, J. J. Kim, H.-Y. Lee, T. Kawai, and H. Y. Choi, Phys. Rev. Lett. 87, 198102 (2001).

${ }^{10}$ J. S. Hwang, K. J. Kong, D. Ahn, G. S. Lee, D. J. Ahn, and S. W. Hwang, Appl. Phys. Lett. 81, 1134 (2002).

${ }^{11}$ B. Q. Xu, P. M. Zhang, X. L. Li, and N. J. Tao, Nano Lett. 4, 1105 (2004)

${ }^{12}$ H. Cohen, C. Nogues, R. Naaman, and D. Porath, Proc. Natl. Acad. Sci. U.S.A. 102, 11589 (2005).

${ }^{13}$ E. Braun, Y. Eichen, U. Sivan, and G. Ben-Yoseph, Nature (London) 391, 775 (1998).

${ }^{14}$ A. J. Storm, J. van Noort, S. de Vries, and C. Dekker, Appl. Phys. Lett. 79, 3881 (2001).
${ }^{15}$ E. Maciá, Phys. Rev. B 76, 245123 (2007).

${ }^{16}$ D. H. Dunlap and V. M. Kenkre, Phys. Lett. A 127, 438 (1988).

${ }^{17}$ F. Bloch, Z. Phys. 52, 555 (1928).

${ }^{18}$ C. Zener, Proc. R. Soc. London, Ser. A 145, 523 (1934)

${ }^{19}$ L. Esaki and R. Tsu, IBM J. Res. Dev. 14, 61 (1970).

${ }^{20}$ N. W. Ashcroft and N. D. Mermin, Solid State Physics (Saunders, New York, 1976), p. 213.

${ }^{21}$ J. Feldmann, K. Leo, J. Shah, D. A. B. Miller, J. E. Cunningham, T. Meier, G. von Plessen, A. Schulze, P. Thomas, and S. Schmitt-Rink, Phys. Rev. B 46, R7252 (1992).

${ }^{22}$ K. Leo, P. Haring Bolivar, F. Brüggemann, R. Schwedler, and K. Köhler, Solid State Commun. 84, 943 (1992).

${ }^{23}$ K. Leo, Semicond. Sci. Technol. 13, 249 (1998).

${ }^{24}$ M. Ben Dahan, E. Peik, J. Reichel, Y. Castin, and C. Salomon, Phys. Rev. Lett. 76, 4508 (1996).

${ }^{25}$ S. R. Wilkinson, C. F. Bharucha, K. W. Madison, Q. Niu, and M. G. Raizen, Phys. Rev. Lett. 76, 4512 (1996).

${ }^{26}$ B. P. Anderson and M. A. Kasevich, Science 282, 1686 (1998).

${ }^{27}$ G. von Plessen, T. Meier, J. Feldmann, E. O. Gobel, P. Thomas, K. W. Goossen, J. M. Kuo, and R. F. Kopf, Phys. Rev. B 49, 14058 (1994).

${ }^{28}$ V. D. Lakhno and N. S. Fialko, Pis'ma Zh. Eksp. Teor. Fiz. 79, 575 (2004).

${ }^{29}$ M. Peyrard and A. R. Bishop, Phys. Rev. Lett. 62, 2755 (1989).

${ }^{30}$ S. Komineas, G. Kalosakas, and A. R. Bishop, Phys. Rev. E 65, 061905 (2002).

${ }^{31}$ P. Maniadis, G. Kalosakas, K. Ø. Rasmussen, and A. R. Bishop, Phys. Rev. E 72, 021912 (2005).

${ }^{32}$ J. A. Berashevich, A. D. Bookatz, and T. Chakraborty, J. Phys.: Condens. Matter 20, 035207 (2008). 